\title{
EFEITOS DO CONTROLE NAS INTENÇÕES COMPORTAMENTAIS DOS CONSUMIDORES INSATISFEITOS
}

THE EFFECTS OF CONTROL ON DISSATISFIED CONSUMERS' BEHAVIORAL INTENTIONS EFECTOS DEL CONTROL EN LAS INTENCIONES COMPORTAMENTALES DE LOS CONSUMIDORES

INSATISFECHOS

\section{RESUMO}

O controle tem sido citado como elemento essencial para o desenvolvimento de interações interpessoais satisfatórias, estando positivamente relacionado à satisfação e a respostas afetivas e comportamentais positivas na área do comportamento do consumidor. Este trabalho investiga o impacto dos controles comportamental, cognitivo e de decisão, representados respectivamente pelas variáveis coprodução, informação e opção de reembolso, nas intenções de recompra e de boca-a-boca positivo após compras insatisfatórias. Os resultados de dois estudos experimentais conduzidos indicam que coprodução e opção de reembolso são capazes de elevar os níveis de intenção de recompra e de boca-a-boca positivo após episódios de insatisfação, enquanto a informação não tem efeito nas intenções comportamentais, mas modera o efeito da coprodução nas intenções comportamentais.

PALAVRAS-CHAVE Controle, coprodução, informação, reembolso, insatisfação.

Natália Araujo Pacheco natipa@brturbo.com.br

Doutoranda em Administração de Empresas pela Escola de Administração, Universidade Federal do Rio Grande do Sul - Porto Alegre - RS, Brasil

Cristiane Pizzutti dos Santos cpsantos@ea.ufrgs.br

Professora da Escola de Administração, Universidade Federal do Rio Grande do Sul - Porto Alegre - RS, Brasil

Renaud Lunardo renaud.lunardo@bem.edu

Professor da Bordeaux Ecole de Management - Talence Cedex, France

Abstract Control has been cited as an essential element for developing satisfactory interpersonal relationships, positively related to satisfaction and to emotional and behavioral responses in consumer behavior. This work investigates the impact of behavioral, cognitive and decisional controls, represented by co-production, information and refund choice, respectively, on repurchase and positive word of mouth intentions after unsatisfactory purchases. The results from two experimental studies indicate that co-production and refund choice lead to higher repurchase and positive word-of-mouth intentions after dissatisfaction episodes, while information do not affect behavioral intentions but moderates the effect of co-production on behavioral intentions.

keywordsControl, co-production, information, refund, dissatisfaction.

Resumen El control ha sido citado como elemento esencial para el desarrollo de interacciones interpersonales satisfactorias, estando positivamente relacionado a la satisfacción y a respuestas afectivas y comportamentales positivas en el área del comportamiento del consumidor. Este trabajo investiga el impacto de los controles comportamental, cognitivo y de decisión, representados respectivamente por las variables coproducción, información y opción de reembolso, en las intenciones de recompra y de boca a boca positivo después de compras insatisfactorias. Los resultados de dos estudios experimentales conducidos indican que coproducción y opción de reembolso son capaces de elevar los niveles de intención de recompra y de boca a boca positivo después de episodios de insatisfacción, mientras que la información no tiene efecto en las intenciones comportamentales, pero modera el efecto de la coproducción en las intenciones comportamentales.

Palabras clave Control, coproducción, información, reembolso, insatisfacción. 


\section{INTRODUÇÃO}

Citado como elemento essencial para o desenvolvimento de interações interpessoais satisfatórias, o controle tem sido definido como a necessidade de se demonstrar competência, superioridade e domínio sobre o ambiente (WHITE, 1959). Neste sentido, tem-se destacado o importante papel do controle na vida das pessoas e as implicações negativas causadas na sua ausência. Averill (1973), por exemplo, afirma que a falta de controle é uma condição que gera estresse. Bateson (1985) argumenta que eventos estressantes que sejam previsíveis ou controláveis terão impacto negativo menor na saúde de um indivíduo.

Averill (1973) afirma que controle tem sido investigado sob três aspectos: comportamental, cognitivo e de decisão. Controle comportamental envolve a influência ou a ação direta no ambiente, controle cognitivo refere-se à interpretação e à avaliação de um indivíduo sobre um evento, assim como também à previsibilidade associada ao ganho de informação que esse indivíduo possa ter, enquanto controle de decisão representa a oportunidade de escolher entre diferentes cursos de ação (AVERILL, 1973). Sua tipologia tem sido largamente citada em trabalhos que abordam o tema (e.g., BATESON, 1985; FARANDA, 2001; HUI; BATESON, 1991; HUI; TOFFOLI, 2002; VAN ROMPAY e outros, 2008).

Com base na tipologia apresentada, este artigo considera a coprodução, que é a participação do consumidor nas atividades de produção de um bem físico ou serviço (ETGAR, 2008), uma forma de prover controle comportamental ao consumidor, visto que a coprodução envolve ações do consumidor que modificam as características do produto ofertado. Da mesma forma, também considera o fornecimento de informações ao consumidor em relação ao produto a ser comprado uma forma de prover controle cognitivo, visto que esse tipo de informação pode trazer a previsibilidade citada por Averill (1973) como elemento chave do controle cognitivo. Por fim, considera-se o oferecimento de opção de reembolso ao consumidor, isto é, a possibilidade de devolver um produto e pedir o reembolso do dinheiro despendido na compra, uma forma de prover controle de decisão a esse consumidor, pois representa uma opção de escolha.

O presente artigo investiga os efeitos das variáveis coprodução, fornecimento de informação e opção de reembolso, representantes dos três tipos de controle identificados por Averill (1973), em dois tipos de in- tenções comportamentais do consumidor - intenção de recompra e intenção de boca-a-boca positivo - após compras insatisfatórias. A decisão de se estudarem as intenções comportamentais após resultados negativos deve-se ao fato de essas situações serem capazes de gerar consequências negativas às empresas, uma vez que sentimentos de insatisfação por parte do consumidor estão associados a respostas comportamentais desfavoráveis, tais como a troca do provedor de serviços, o boca-a-boca negativo (SINGH, 1988, FERNANDES e SANTOS, 2008) e comportamentos de retaliação frente à empresa (HUEFNER e HUNT, 2000). Busca-se, assim, investigar fatores que podem mitigar essas respostas desfavoráveis.

Este artigo, portanto, busca responder ao seguinte questionamento: a coprodução, a disponibilidade de informações e a possibilidade de reembolso podem atenuar os efeitos negativos causados pela insatisfação do consumidor com uma compra? Esse questionamento é relevante à medida que poderá mostrar às empresas que oferecer ao consumidor a possibilidade de coproduzir, de ter mais informações e a opção de reembolso pode funcionar como uma espécie de "amortecedor" dos efeitos negativos da insatisfação nas intenções de recompra e de boca-a-boca positivo deste consumidor, devido ao aumento de sua percepção de controle sobre o processo de produção ou de compra do produto.

Para responder a esse questionamento foram realizados dois estudos experimentais, que buscam analisar os efeitos individuais e conjunto das variáveis associadas ao controle nas intenções de recompra e de boca-a-boca positivo após episódios de compras insatisfatórios (também chamados nesse trabalho de resultados negativos), sendo que no estudo 1 manipula-se coprodução e informação, enquanto que no estudo 2 manipula-se coprodução e opção de reembolso.

Ressalta-se que a investigação proposta torna-se ainda mais relevante uma vez que a interação entre consumidor e empresa tem sido apontada como fonte de criação de valor (PRAHALAD e RAMASWAMY, 2004; VARGO e LUSCH, 2008), de obtenção de valor econômico (CHAN, YIM, LAM, 2010) e de eficácia competitiva (BENDAPUDI e LEONE, 2003), o que faz da coprodução uma ferramenta de marketing eficaz do ponto de vista da empresa. A literatura sobre coprodução, no entanto, tem-se limitado a investigar a satisfação do consumidor como resultante desse processo (e.g., BENDAPUDI e LEONE, 2003; ORDANINI e PASINI, 2008; CHAN, YIM, LAM, 2010; HUNT, ONETO, VARCA, 2010), enquanto negligencia intenções comportamentais importantes, 
tais como recompra e boca-a-boca positivo. Da mesma forma, não há pesquisas que tenham explorado os efeitos da coprodução em casos de resultados insatisfatórios, com exceção do trabalho de Bendapudi e Leone (2003), que investiga o efeito de resultados piores que os esperados na satisfação do consumidor.

O restante do texto está organizado da seguinte forma: primeiro apresenta-se a fundamentação teórica e a formulação das hipóteses sobre os efeitos da coprodução, da informação e da opção de reembolso, fontes de controle comportamental, cognitivo e de decisão, nas intenções de recompra e de boca-a-boca positivo em episódios de insatisfação. Em seguida, explica-se o método do estudo 1 e seus resultados, fazendo-se o mesmo para o estudo 2. Por fim, são apresentadas as conclusões e limitações deste trabalho.

\section{COPRODUÇÃO, INFORMAÇÃO E OPÇÃO DE REEMBOLSO COMO FONTES DE CONTROLE}

Controle é a habilidade percebida para alterar significativamente uma situação (BURGER, 1989; THOMPSON, 1981) ou, de forma semelhante, a crença de que um indivíduo pode influenciar seu ambiente e/ou trazer os resultados desejados (VAN RAAIJ e PRUYN, 1998). O controle vem sendo tratado como elemento importante na vida das pessoas, cuja ausência pode gerar estresse (AVERILL, 1973) e cuja presença é associada a consequências positivas, como satisfação e desempenho superior no trabalho (e.g. GREENBERGER e outros, 1989; BOND e BUNCE, 2003). Na área de comportamento do consumidor, Chang (2008) também encontrou relação positiva entre controle e satisfação, ao estudar situações de recuperação de serviço, enquanto que Hui e Bateson (1991) verificaram que a percepção de controle impacta positivamente nas respostas afetivas (prazer) e, consequentemente, comportamentais (aproximação) do consumidor. Dentre as diferentes tipologias de controle existentes, a tipologia de controle de Averill (1973) tem sido amplamente reconhecida por pesquisadores (e.g., BATESON, 1985; FARANDA, 2001; HUI e BATESON, 1991; HUI e TOFFOLI, 2002; SKINNER, 1996; VAN ROMPAY e outros, 2008), apresentando três tipos de controle: comportamental, cognitivo e de decisão.
O controle comportamental refere-se a uma resposta que pode influenciar diretamente ou modificar as características de um evento (AVERILL, 1973). Ao fazer um estudo integrativo dos diversos construtos relacionados a controle, Skinner (1996) relaciona esse tipo de controle ao indivíduo que modifica um resultado através de suas ações ou comportamentos. Averill (1973) destaca que o controle comportamental envolve ação direta no ambiente, enquanto o controle cognitivo refere-se à forma como um evento é interpretado. $\mathrm{O}$ autor subdivide o controle cognitivo em dois tipos: ganho de informação e estimativa, explicando que o controle cognitivo através da informação envolve sinais que possibilitam a previsão de um evento. Bateson (1985), ao analisar esse tipo de controle, também reconhece que o ganho de informação está relacionado à previsibilidade e à antecipação de um evento. $\mathrm{O}$ segundo tipo envolve a imposição de significados aos eventos, o que é visto como um mecanismo de defesa na literatura psicanalítica. Já o controle de decisão é definido como "a variedade de escolha ou número de opções abertas a um indivíduo" (AVERILL, 1973, p. 298) ou, em outras palavras, "a oportunidade de escolher entre vários cursos de ação” (AVERILL, 1973, p. 287).

Com base nessa tipologia, coprodução pode ser considerada uma forma de prover controle comportamental, uma vez que a coprodução implica a influência direta do consumidor nos resultados do processo de produção (ETGAR, 2008). A coprodução é definida como a participação do consumidor nas atividades de produção, sejam elas o desenho de um produto, a agregação de recursos ou outras atividades do processo produtivo que geram um resultado (bem físico ou serviço) que será consumido (ETGAR, 2008).

Além da associação entre coprodução e controle feita a partir da tipologia de Averill (1973) - isso é, coprodução sendo considerada uma representação do controle comportamental -, existe outro aspecto importante envolvendo a coprodução: nela, a satisfação com o processo que gera o produto final pode ser maior, uma vez que o consumidor participa desse processo e pode influenciar o resultado. Uma maior satisfação com o processo de produção, que é diferente da satisfação com o resultado, isso é, a satisfação obtida após a compra (ZHANG e FITZSIMONS, 1999), pode levar a maiores níveis de intenções comportamentais positivas, independentemente do resultado de a compra ser positivo ou negativo, uma vez que satisfação e intenção de recompra estão positivamente relacionadas (CRONIN, BRADY, HULT, 2000; WESTBROOK, 1987), assim como 
também satisfação e boca-a-boca positivo (ATHANASSOPOULOS e outros, 2001; LADHARI, 2007). Com base em Zeithaml, Berry e Parasuraman (1996), intenções comportamentais, nesse trabalho, englobam intenções de recompra e intenções de boca-a-boca positivo, isso é, intenções de falar coisas positivas sobre a empresa para amigos e familiares. O efeito positivo da coprodução na satisfação já foi proposto por Ertimur (2008) e empiricamente verificado por Hunt, Oneto e Varca (2010), no entanto, a literatura não aborda o efeito positivo da coprodução nas intenções comportamentais. Com isso, propõe-se que:

H1a: Após um resultado negativo, consumidores que coproduzem exibirão maior intenção de recompra quando comparados aos consumidores que não coproduzem.

H1b: Após um resultado negativo, consumidores que coproduzem exibirão maior intenção de realizar boca-a-boca positivo quando comparados aos consumidores que não coproduzem.

Além da coprodução, outra variável que também confere controle ao consumidor e que pode influenciar suas intenções comportamentais após compras insatisfatórias merece ser analisada: a informação. A evolução do marketing para uma filosofia "market with", na qual se entende que o consumidor colabora com a empresa no processo de criação de valor (LUSCH, VARGO, O'BRIEN, 2007), torna a informação ainda mais importante para o consumidor, representando uma ferramenta que este poderá utilizar para cocriar valor com a empresa, ou seja, utilizando as informações sobre o produto, o consumidor consegue colaborar com a empresa na criação da oferta mais adequada às suas necessidades e expectativas e também consegue usufruí-la de maneira a aperfeiçoar sua experiência de consumo. De acordo com Dholakia, Zwick e Denegri-Knott (2010), a informação domina a criação de valor na medida em que a manipulação da informação por parte dos consumidores transforma essa informação em valor. Averill (1973) classificou o ganho de informação como um tipo de controle cognitivo, relacionado a sinais que possibilitam a previsão de um evento. A informação leva à previsibilidade, e a habilidade de prever consequências de um evento pode provocar sensação de controle (STAUB, TURSKY, SCHWARTZ, 1971). O efeito da previsibilidade no controle percebido foi verificado por Holt (1995), que mostrou que espectadores de baseball que se sentiam capazes de prever ações no campo percebiam estar mais envolvidos na produção do jogo e tendo algum controle sobre ele.

No que diz respeito aos encontros de serviço, Bateson (1985) afirma que, se o encontro seguir um roteiro que é conhecido por clientes e funcionários e no qual todos saibam quais são os papéis que devem desempenhar, ele torna-se previsível, de maneira muito semelhante ao controle cognitivo por meio de ganho de informação. Assim, clientes e funcionários podem não ter controle direto sobre o encontro, mas um alto nível de informação que garanta a eles certa previsibilidade pode lhes oferecer sensação de controle.

Se o controle é maior quando se tem mais informação, no caso de resultados insatisfatórios, consumidores que tiveram mais informações durante a compra exibirão maiores níveis de intenções comportamentais positivas devido a essa sensação de controle. Outra razão para que isso aconteça é que, quando o consumidor tem mais informações numa situação de compra, isto pode aumentar sua satisfação com o processo, que, por sua vez, aumentaria as intenções comportamentais positivas (CRONIN, BRADY, HULT, 2000; LADHARI, 2007). Considerando esses argumentos, as seguintes hipóteses são formuladas:

H2a: Após um resultado negativo, consumidores que recebem mais informações exibirão maior intenção de recompra quando comparados aos consumidores que recebem menos informações.

H2b: Após um resultado negativo, consumidores que recebem mais informações exibirão maior intenção de realizar boca-a-boca positivo quando comparados aos consumidores que recebem menos informações.

Até o momento, foram estabelecidas relações entre coprodução (representando controle comportamental) e intenções comportamentais positivas, assim como também entre informação (representando controle cognitivo) e as mesmas intenções comportamentais, após episódios de insatisfação. A próxima variável a ser analisada é a opção de reembolso, representando controle de decisão, e sua relação com intenções de recompra e de boca-a-boca positivo, após compras de resultado negativo.

O controle de decisão apresentado na tipologia de Averill (1973) está intimamente relacionado às escolhas de um indivíduo. A teoria de causalidade pessoal de deCharms (1968) argumenta que indivíduos que admitem que seu comportamento é decorrente de suas próprias escolhas percebem maior controle e, consequentemen- 
te, são mais motivados a desempenhar tarefas do que indivíduos que enxergam seu comportamento como um resultado de forças externas. Tal suposição de que as escolhas levam a um maior controle percebido foi empiricamente suportada na literatura (HUI e BATESON, 1991; MILLS e KRANTZ, 1979). Langer (1975) encontrou resultados semelhantes ao pesquisar ilusão de controle em vez de controle percebido. A oportunidade de escolha fez com que indivíduos percebessem maior controle mesmo quando participavam de uma tarefa que dependia unicamente da sorte. Chang (2008) pesquisou a relação entre escolha, controle e satisfação, identificando que a possibilidade de escolha entre opções de recuperação de serviço aumentou a percepção de controle de clientes e sua satisfação com a experiência.

A escolha é algo desejável aos consumidores, uma vez que restrições de escolha podem gerar respostas emocionais como raiva e ressentimento, além de respostas comportamentais que podem variar desde conformidade e adaptação até rejeição e rebelião (BOTTI e outros, 2008). Este trabalho foca nos benefícios que a escolha pode trazer, tais como o aumento da percepção de controle (HUI, BATESON, 1991; MILLS e KRANTZ, 1979) e da satisfação (BOTTI e MCGILL, 2006). Portanto, conceder aos consumidores uma escolha como devolver um produto e pedir reembolso do dinheiro que pagou por ele pode amenizar possíveis reações negativas após uma compra insatisfatória, devido ao aumento de controle de decisão. Se os consumidores percebem ter essa opção de devolução do produto e pedido de reembolso, sua satisfação com o processo provavelmente é maior, fato que também contribui para a presença de intenções comportamentais positivas mais elevadas. Com base nisso, as próximas hipóteses são propostas:

H3a: Após um resultado negativo, consumidores que possuem opção de reembolso exibirão maior intenção de recompra quando comparados aos consumidores que não possuem opção de reembolso.

H3b: Após um resultado negativo, consumidores que possuem opção de reembolso exibirão maior intenção de realizar boca-a-boca positivo quando comparados aos consumidores que não possuem opção de reembolso.

Uma vez estabelecidas as relações entre intenções comportamentais e coprodução, informação e opção de reembolso, os efeitos de interação entre essas três variáveis independentes também podem ser investigados. As empresas podem oferecer a seus clientes a possibilidade de coprodução, diferentes níveis de informação e opção de reembolso conforme julgarem conveniente. Torna-se desejável saber se a combinação dessas três ferramentas gerenciais propicia resultados mais vantajosos que adotá-las separadamente. De acordo com Cornelius e Averill (1980), enquanto o controle comportamental reduz o estresse na presença de informação (controle cognitivo), ele pode também aumentar o estresse na ausência da informação. Dessa forma, o consumidor que tem a chance de coproduzir um bem físico ou serviço, ou seja, o consumidor que tem controle comportamental, terá seu estresse reduzido no processo de produção e compra se um alto nível de informação estiver disponível, uma vez que informação pode trazer previsibilidade (AVERILL, 1973; BATESON, 1985) e pode fazer com que o consumidor sinta-se mais capaz de realizar a tarefa exigida pela coprodução. No entanto, se é dado ao consumidor a possibilidade de coproduzir, mas não forem fornecidas informações para que ele o faça de forma adequada, isso reduz os efeitos positivos da coprodução e aumenta o estresse com o processo de produção. Assim, o consumidor que coproduz e que recebe alto nível de informação demonstrará maiores níveis de intenções comportamentais positivas após um resultado insatisfatório em comparação a um consumidor que recebe baixo nível de informação (i.e., maior nível de informação disponibilizado ao consumidor potencializará o efeito da coprodução). As seguintes hipóteses são, então, formuladas:

H4a: Após um resultado negativo, o nível de informação disponível moderará o efeito da coprodução na intenção de recompra, sendo que este efeito será mais forte (fraco) quando houver alto (baixo) nível de informação disponível.

H4b: Após um resultado negativo, o nível de informação disponível moderará o efeito da coprodução na intenção de realizar boca-a-boca positivo, sendo que este efeito será mais forte (fraco) quando houver alto (baixo) nível de informação disponível.

Cornelius e Averill (1980) afirmam que diferentes tipos de controle podem interagir de forma complexa, como no caso da interação entre controle comportamental e cognitivo, ou podem ter efeitos independentes, como no caso do controle de decisão, que não teve interação com o controle comportamental em seu estu- 
do. Para o presente trabalho, ao se recorrer à literatura sobre o tema, não foi encontrada fundamentação teórica que permitisse a formulação de uma hipótese de interação entre a coprodução, representante do controle comportamental, e a opção de reembolso, representante do controle de decisão. Se por um lado o estudo de Cornelius e Averill (1980) indica não haver interação entre os efeitos destes dois tipos de controle, por outro lado, Witt, Andrews e Kacmar (2000) verificaram que, no ambiente organizacional, a presença de políticas organizacionais, que, pressupõe-se, acarretariam a diminuição do controle comportamental dos funcionários, reduzem a satisfação desses com o trabalho, sendo esse efeito mais fraco entre funcionários que participam da tomada de decisão com seus supervisores. Os resultados desse estudo sinalizam, assim, que poderia haver interação entre controle comportamental e de decisão. Dessa forma, mesmo não existindo base teórica suficiente para se formular uma hipótese de interação entre esses dois tipos de controle, propõe-se nesse estudo a investigação sobre a relação entre coprodução e opção de reembolso em caráter exploratório (i.e., sem a formulação e teste de hipótese). Essa investigação torna-se relevante à medida que ajudará a compreender se clientes que coproduzem e, consequentemente, possuem controle comportamental sentem ainda maior controle sobre a compra se também tiverem a opção de pedir reembolso (controle de decisão). Em outras palavras, a investigação a ser feita é se a opção de reembolso intensificaria o efeito da coprodução nas intenções comportamentais, em episódios de insatisfação, de forma semelhante ao que ocorreria entre coprodução e informação.

Para investigação das hipóteses formuladas, foram conduzidos dois estudos experimentais. O primeiro estudo manipulou coprodução e informação no contexto de serviços para examinar seus efeitos nas intenções de recompra e de boca-a-boca positivo após um resultado negativo. O segundo estudo manteve os mesmos objetivos do primeiro, manipulando coprodução e opção de reembolso na compra de um bem físico. Na sequência, são apresentadas a descrição dos procedimentos metodológicos e dos resultados encontrados em cada estudo experimental e a discussão geral.

\section{ESTUDO 1}

O primeiro estudo utilizou um desenho experimental 2 (coprodução: com e sem) x 2 (nível de informação: alto e baixo). Participaram voluntariamente desse estudo 97 estudantes de uma universidade brasileira, que foram aleatoriamente designados a um dos cenários do experimento. Coprodução e informação foram manipuladas através de cenários escritos que simulavam a compra de uma viagem de férias.

Os participantes receberam um questionário com a seguinte narrativa: "Depois de um difícil semestre na universidade, você decidiu viajar de férias para o nordeste brasileiro e vai a uma agência de viagens para conferir as possibilidades". A partir deste ponto, a sequência da narrativa variava para manipular a coprodução e a informação. Participantes que receberam o cenário de coprodução leram o seguinte texto: "Quando você chega à agência, o atendente explica que você pode montar seu próprio roteiro de viagem para o Nordeste, assim sua viagem terá exatamente as características que você desejar". Participantes do cenário sem coprodução receberam o texto: "Quando você chega à agência, o atendente explica que pode lhe oferecer três opções de pacotes para o Nordeste e que não é possível fazer um pacote diferente destes". Cenários de baixa informação apresentaram o texto: "O atendente lhe passa informações sobre preços. Mais nenhuma informação lhe é oferecida”. Já os cenários de alto nível de informação continham o texto: "O atendente da agência lhe fornece um livreto onde você encontra informações sobre cada cidade dessa região do país, tais como pontos turísticos, eventos culturais e festas. Você também recebe informações sobre hotéis, pousadas e restaurantes. $\mathrm{O}$ atendente lhe passa informações sobre preços". Após a manipulação da coprodução e da informação, todos os participantes receberam o seguinte texto sobre o resultado da viagem: "Imagine que você fez sua viagem para o Nordeste e que ela foi uma experiência negativa devido às escolhas que você fez na agência de viagens".

Foi conduzido um teste piloto com 89 estudantes antes da efetiva realização do estudo 1 . O teste piloto revelou a eficácia da manipulação da coprodução e indicou a necessidade de melhorias na manipulação da informação. Os cenários acima apresentados já contemplam as melhorias realizadas após apuração dos resultados do teste piloto.

\section{Medidas}

Os participantes receberam um questionário com uma questão para checagem da manipulação da coprodução ("Ajudei a montar o roteiro da viagem que 
comprei") e duas para checagem da manipulação da informação ("Em relação à quantidade de informação fornecida pela agência de viagens, considero ter sido: insuficiente/suficiente; baixo nível de informação/ alto nível de informação"). O questionário também apresentou afirmações com escalas de resposta que variavam de 1 a 7 ( 1 = muito improvável, 7 = muito provável) para mensurar as intenções comportamentais. A intenção de recompra foi medida utilizando uma escala de três itens $(\alpha=0,95)$ adaptada a partir do trabalho de Zeithaml, Berry e Parasuraman (1996): "Qual é a probabilidade de você contratar os serviços dessa agência de viagens novamente?", "Qual é a probabilidade de você fazer negócios com essa agência de viagens no futuro?" e "Qual é a probabilidade de você considerar essa agência de viagens como sua primeira opção para a compra de uma viagem?". A intenção de realizar boca-a-boca positivo foi medida com uma escala de três itens $(\alpha=0,96)$ também adaptada a partir de Zeithaml, Berry e Parasuraman (1996): "Qual é a probabilidade de você recomendar essa agência de viagens para seus amigos e familiares?", "Qual é a probabilidade de você falar coisas positivas sobre essa agência para outras pessoas?" e "Qual é a probabilidade de você encorajar seus amigos e familiares a fazer negócio com essa agência?". Tais escalas já haviam apresentado bons índices de confiabilidade no teste piloto ( $\alpha=0,96$ para intenção de recompra e $\alpha=0,92$ para boca-a-boca positivo).

\section{Resultados e discussão}

Checagem das manipulações: Com base nos resultados alcançados através de uma ANOVA 2 x 2, ambas as manipulações foram eficazes. A percepção de coprodução dos participantes foi significativamente maior nos cenários de coprodução $(M=5,72)$ que nos cenários sem coprodução $(M=3,00 ; F(1,93)=59,37, p<0,01)$, e não houve efeito da informação $(F(1,93)=0,67, p>0,10)$ ou da interação entre coprodução e informação $(F(1$, $93)=0,14, p>0,10)$ sobre ela. A percepção dos participantes sobre o nível de informação foi maior nos cenários de alta informação $(M=5,45)$ que nos cenários de baixa informação $(M=2,50 ; F(1,93)=85,55, p<$ $0,01)$, não ocorrendo efeito da coprodução $(F(1,93)$ $=0,23, p>0,10)$ ou da interação entre coprodução e informação $(F(1,93)=0,12, p>0,10)$ sobre ela.

Intenções comportamentais: A ANOVA revelou o efeito principal significativo da coprodução nas intenções de recompra $(F(1,93)=4,12, p<0,05)$ e de boca- -a-boca positivo $(F(1,93)=4,64, p<0,05)$. Nos cenários de coprodução, intenções de recompra $(M=2,02)$ e de boca-a-boca $(M=1,98)$ foram maiores que em cenários sem coprodução $(M=1,55$, para intenções de recompra e $M=1,48$, para intenções de boca-a-boca), suportando H1a e H1b.

Apesar de não terem sido encontrados até o momento trabalhos que investigassem, especificamente, os efeitos da coprodução em episódios de insatisfação, o fato de os resultados deste estudo apresentarem evidência de que a coprodução pode atenuar os efeitos negativos da insatisfação nas intenções comportamentais do consumidor era esperado, uma vez que coprodução confere ao consumidor controle comportamental nos termos definidos por Averill (1973). Ainda, a literatura apresenta relações positivas entre coprodução e satisfação (BENDAPUDI e LEONE, 2003; HUNT e outros, 2010), entre controle e satisfação (GREENBERGER e outros, 1989; BOND e BUNCE, 2003; CHANG, 2008), e entre controle e respostas afetivas e comportamentais - prazer e aproximação (HUI e BATESON, 1991), o que amenizaria as consequências em episódios de insatisfação.

O nível de informação não teve efeito significativo nas intenções comportamentais $(F(1,93)=1,27, p>$ 0,10 , para intenção de recompra e $F(1,93)=2,92, p>$ 0,05 , para boca-a-boca positivo), assim H2a e H2b não foram suportadas. Tal resultado indica que o controle cognitivo oriundo das informações fornecidas ao cliente não foi suficiente para atenuar os efeitos negativos decorrentes da insatisfação. Apesar de contrário ao que era esperado, tal resultado encontra sustentação teórica no estudo de Skinner (1996). De acordo com esse autor, há evidência de que informação e previsibilidade nem sempre levam a percepções de controle, por isso seria importante o questionamento sobre as condições nas quais ambas as variáveis levariam ao controle. Uma dessas condições parece ter sido evidenciada no efeito de interação significativo da coprodução e da informação obtido neste estudo e discutido a seguir.

Apesar da falta de suporte às hipóteses H2a e H2b, os efeitos interativos da coprodução e da informação nas intenções de recompra $(F(1,93)=5,13, p<0,05)$ e de boca-a-boca $(F(1,93)=4,70, p<0,05)$ foram significativos. Para esclarecer esses efeitos interativos e testar as hipóteses H4a e H4b, o banco de dados do estudo 1 foi dividido em dois, um banco de dados com participantes dos cenários com alto nível de informação disponível e outro com participantes dos cenários com baixo nível de informação disponível, possibilitando a realização de testes post-hoc, através de ANOVA em 
cada um desses bancos. Os resultados mostraram que, entre aqueles expostos a um alto nível de informação, as intenções de recompra $(\mathrm{M}=2,41, F(1,47)=8,13$, $p<0,01)$ e de boca-a-boca positivo ( $\mathrm{M}=2,43, F$ ( 1 , $48)=7,76, p<0,01$ ) foram significativamente maiores quando eles também eram expostos à coprodução, do que quando não eram expostos à coprodução $(M=1,42$, para intenção de recompra; $M=1,43$, para boca-a-boca positivo). Já entre aqueles com baixo nível de informação, as médias das variáveis dependentes não foram significativamente diferentes para aqueles que foram expostos à coprodução $(\mathrm{M}=1,63, F(1,46)=0,03, p>0,1$ para intenções de recompra; $\mathrm{M}=1,53, F(1,46)=0,00$, $p>0,1$ para intenções de boca-a-boca) daquelas encontradas no contexto onde não havia coprodução ( $M=1,68$, para intenção de recompra; $M=1,54$, para boca-a-boca positivo). Assim, evidencia-se que o nível de informação modera o efeito da coprodução, conforme proposto por H4a e H4b, uma vez que o efeito positivo da coprodução nas intenções comportamentais é significativo apenas quando é oferecido alto nível de informação; quando é oferecido baixo nível de informação, os níveis de intenções comportamentais são semelhantes, independentemente se o contexto é de coprodução ou não. Esse resultado indica uma mode-

\section{Gráfico 1 - Intenção de recompra como uma função da coprodução e da informação no estudo 1}
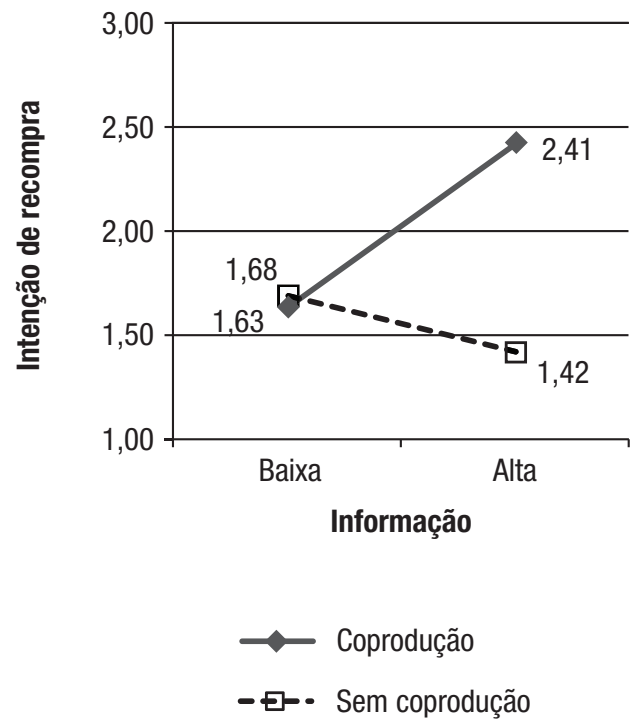

ração total do nível de informação, isto é, ela potencializa o efeito da coprodução quando disponibilizada em altos níveis e reduz esse efeito a zero, quando em baixos níveis. Note que esse resultado não rejeita $\mathrm{H} 1$, mas sim evidencia uma condição limitadora para o efeito da coprodução nas intenções comportamentais.

Complementarmente, dividiu-se o banco de dados geral em outros dois grupos, um com participantes dos cenários com coprodução e outro com participantes dos cenários sem coprodução. Verificou-se através de ANOVA que, para participantes nos cenários com coprodução, maior nível de informação levou a maior intenção de recompra $(\mathrm{M}=2,41, F(1,48)=4,40, p<0,05)$ e de boca-a-boca positivo $(\mathrm{M}=2,43, F(1,48)=5,22$, $p<0,05)$ do que o baixo nível de informação $(M=1,63$ para intenção de recompra; $M=1,53$ para boca-a-boca positivo), enquanto, para participantes nos cenários sem coprodução, o nível de informação (maior ou menor) não alterou as intenções de recompra do consumidor $(F(1,45)=1,00, p>0,10)$ e de boca-a-boca positivo $(F(1,45)=0,21, p>0,10)$, evidenciando que o nível de informação é relevante para o aumento das intenções de recompra e de boca-a-boca positivo apenas em situações nas quais coprodução é oferecida. Esses resultados são apresentados nos gráficos 1 e 2.

\section{Gráfico 2 - Intenção de boca-a-boca como uma função da coprodução e da informação no estudo 1}

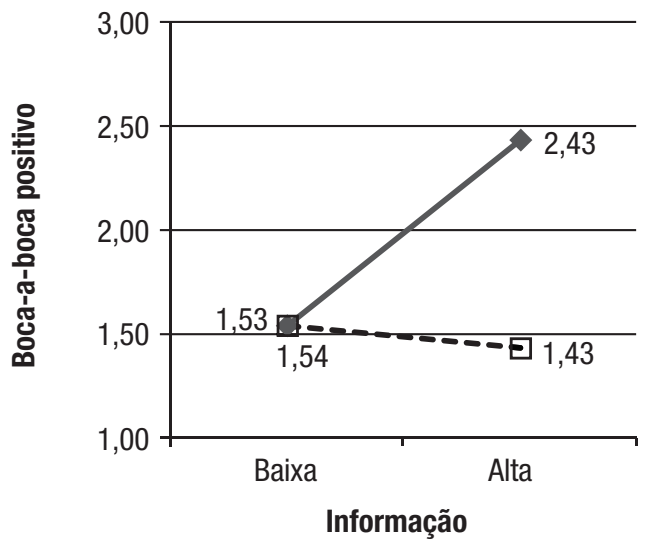


Os resultados apresentados mostram que as intenções de recompra e de boca-a-boca positivo foram relativamente baixas nos cenários de coprodução com alto nível de informação, apesar de serem significativamente maiores que nos cenários nos quais havia alto nível de informação, mas não havia coprodução ou nos quais coprodução era oferecida com baixo nível de informação. Tal resultado não é surpresa, pois as intenções comportamentais foram medidas após uma compra insatisfatória. O relevante nesses resultados é a descoberta de que a presença da coprodução juntamente com a informação gera maiores níveis de intenções comportamentais quando comparada a cenários nos quais tais variáveis estão ausentes.

Resultados do estudo 1 mostram que, mesmo quando os consumidores enfrentam uma experiência negativa, a coprodução eleva as intenções de recompra e de realização de boca-a-boca positivo. Porém esse efeito é encontrado apenas quando juntamente com a coprodução é fornecido alto nível de informação aos consumidores. Além disso, simplesmente oferecer um maior nível de informação sem associá-lo à coprodução não teve impacto positivo nas intenções comportamentais; portanto a previsibilidade oriunda da informação não foi suficiente para minimizar os efeitos negativos da experiência de insatisfação deste estudo. Dessa forma, no presente estudo, a informação é considerada uma variável moderadora pura, conforme tipologia de Sharma, Durand e Gur-Arie (1981), pois influenciou a força da relação entre coprodução e intenções comportamentais, através de uma interação significativa com a variável preditora (i.e., coprodução), mas não impactou diretamente nas variáveis de critério (ou dependentes, i.e., intenções comportamentais). Esse resultado não invalida ou rejeita a relação encontrada entre coprodução e intenções comportamentais sem a consideração dessa variável. Assim, tanto o efeito principal da coprodução nas variáveis dependentes como também o efeito interativo com a informação estão propostos e suportados pelos resultados do estudo.

O estudo 2 estende a investigação do primeiro estudo para um contexto de coprodução de um bem físico, e baseia-se na proposta de que a coprodução levará a maiores níveis de intenções de recompra e de boca-a-boca positivo após um resultado insatisfatório e na proposta de que a opção de reembolso também levará a maiores níveis dessas intenções comportamentais.

\section{ESTUDO 2}

Semelhante ao estudo 1, o segundo experimento teve um desenho 2 (coprodução: com e sem) x 2 (opção de reembolso: com e sem). Noventa (90) estudantes de uma universidade francesa participaram dele. Foram adotados cenários escritos novamente, desta vez para manipular coprodução e opção de reembolso na simulação da compra de uma escrivaninha. O objetivo de desenvolver o segundo estudo com um produto diferente e com estudantes de outra nacionalidade foi aumentar a validade externa dos efeitos da coprodução encontrados no primeiro estudo. Todavia, não foram feitos testes comparando as duas amostras, pois esse não era o foco do trabalho.

Os participantes receberam a seguinte narrativa: "Você comprou um computador para desenvolver suas atividades pessoais e profissionais e agora você precisa de uma escrivaninha na qual você possa mantê-lo. Você vai a uma loja para olhar alguns modelos de escrivaninha que estão lá expostos". Os participantes que foram aleatoriamente designados a cenários de coprodução leram na sequência o texto: "Você pergunta ao vendedor se poderia desenhar sua própria escrivaninha. O vendedor responde que é possível e chama um funcionário que prontamente começa a desenhá-la seguindo suas instruções. Desta forma, sua escrivaninha terá as características (altura, largura, divisões, acessórios etc.) que você desejar”. Participantes do cenário sem coprodução receberam um texto diferente: "Você pergunta ao vendedor se poderia desenhar sua própria escrivaninha. O vendedor diz que não é possível, pois a fábrica produz apenas escrivaninhas idênticas às expostas na loja. Então você continua olhando os modelos da loja". Com o intuito de manipular a opção de reembolso, dois textos distintos foram fornecidos aos participantes, um contendo a opção de reembolso: "O vendedor informa que a loja possui uma política de satisfação garantida, a qual oferece reembolso integral no caso de devolução do produto", e outro sem essa opção: "O vendedor informa que a loja não possui uma política de satisfação garantida, o que significa que você não será reembolsado no caso de devolução do produto". Todos os participantes, independentemente do cenário recebido, foram informados de que realizaram a compra e também receberam a narrativa: "Imagine que você não gostou da escrivaninha que você comprou”.

É importante ressaltar que o fato de a informação disponível não ter sido manipulada aqui, isso é, não 
ter sido explicitado ao respondente se ele recebeu informações da empresa ou não, não significa que os cenários desse segundo estudo possam ser considerados cenários com pouca informação, o que resultaria, segundo os achados do estudo 1, em cenários onde a coprodução não teria efeito significativo nas intenções comportamentais. Assim, espera-se que H1, que propõe a relação entre coprodução e intenções comportamentais, seja confirmada também nesse segundo experimento, além de se investigar, ainda que de forma exploratória, o papel da opção de reembolso como possível moderadora dessa relação.

\section{Medidas}

A checagem da manipulação da coprodução foi feita através da questão "Ajudei a desenhar a escrivaninha que comprei", enquanto a da opção de reembolso foi feita através da questão "A loja oferece-me a possibilidade de ser reembolsado caso não esteja satisfeito". As mesmas escalas do estudo 1 foram utilizadas para medir intenções de recompra $(\alpha=.88)$ e de boca-a-boca $(\alpha=.95)$.

\section{Resultados e discussão}

Checagem da Manipulação: A estimativa de coproduÇão dos participantes foi significativamente maior em cenários de coprodução $(M=5,98)$ que em cenários sem coprodução $(M=1,41, F(1,86)=455,12, p<0,01)$, não sendo influenciada pela opção de reembolso $(F(1,86)=1,44, p>0,10)$ ou pela interação entre coprodução e opção de reembolso $(F(1,86)=1,01, p>$ $0,10)$, indicando que a manipulação da coprodução foi eficaz. Do mesmo modo, a percepção de opção de reembolso foi maior em cenário com opção $(M=6,09)$ do que em cenários sem opção de reembolso ( $M=1,98$, $F(1,86)=151,21, p<0,01)$ e não houve influência da coprodução $(F(1,86)=0,13, p>0,10)$ ou da interação entre coprodução e opção de reembolso $(F(1$, 86) $=0,11, p>0,10)$.

Intenções comportamentais: Os resultados obtidos para coprodução no estudo 2 foram similares aos do estudo 1. A ANOVA revelou efeitos significativos da coprodução nas intenções de recompra $(F(1,86)=$ $12,79, p<0,01)$ e de boca-a-boca $(F(1,86)=10,29$, $p<0,01)$. Como previsto nas hipóteses, as intenções de recompra e de boca-a-boca positivo foram maiores em cenário de coprodução $(M=2,54$, para intenções de recompra, $M=2,70$, para intenções boca-a-boca) do que em cenários sem coprodução ( $M=1,84$, para intenções de recompra, $\mathrm{M}=1,93$, para intenções de boca-a-boca). Esses resultados suportam H1a e H1b.

A opção de reembolso também teve efeito significativo nas intenções de recompra $(F(1,86)=9,25, p$ $<0,01)$ e de boca-a-boca $(F(1,86)=7,98, p<0,05)$, sendo que os cenários com essa opção contaram com maiores níveis de intenções comportamentais dos participantes ( $M=2,49$, para intenções de recompra, $M=2,64$, para intenções de boca-a-boca) do que os cenários sem opção de reembolso ( $M=1,90$, para intenções de recompra e $\mathrm{M}=1,98$, para intenções de boca-a-boca). Esses resultados suportam $\mathrm{H} 3 \mathrm{a}$ e $\mathrm{H} 3 \mathrm{~b}$. Tal resultado da opção de reembolso nas intenções comportamentais do consumidor insatisfeito corrobora outros estudos que mostram a possibilidade de escolha como algo positivo, que aumenta a percepção de controle (HUI e BATESON, 1991; MILLS e KRANTZ, 1979) e a satisfação (BOTTI e MCGILL, 2006). O fato de participantes que não tiveram a opção de reembolso apresentarem níveis mais baixos de intenções comportamentais está em linha com o estudo de Botti e outros (2008), que mostra que restrições em relação à escolha podem gerar respostas comportamentais negativas.

Não houve efeito de interação significativo da coprodução e da opção de reembolso nas intenções de recompra $(F(1,86)=0,22, p>0,05)$ e de boca-a-boca $(F(1,86)=0,28, p>0,05)$. Os Gráficos 3 e 4 mostram esse resultado. Semelhante ao estudo 1 , as intenções comportamentais foram relativamente baixas na presença das variáveis independentes, porém foram maiores que nos cenários nos quais coprodução e opção de reembolso estavam ausentes.

Os resultados encontrados para coprodução no estudo 2 reafirmam aqueles encontrados no estudo 1 , mostrando que esta pode minimizar os efeitos de uma compra insatisfatória nas intenções comportamentais. Os resultados do estudo 2 mostram, ainda, que conceder a opção de solicitar reembolso na compra de um produto também eleva as intenções comportamentais após um resultado insatisfatório, porém a falta de efeito significativo da interação entre coprodução e opção de reembolso rejeita a ideia de que oferecer a opção de reembolso potencializaria os efeitos da coprodução nas intenções comportamentais, convergindo com os resultados encontrados por Cornelius e Averill (1980), os quais indicam que os controles de decisão e comportamental possuem efeitos independentes. Os efeitos individuais da coprodução e da opção de reembolso, representantes respectivamente dos controles compor- 
tamental e de decisão, convergem com os resultados positivos encontrados na literatura sobre controle em relação à satisfação (GREENBERGER e outros, 1989; BOND e BUNCE, 2003; CHANG, 2008), e a respostas afetivas e comportamentais positivas (HUI e BATESON, 1991), sugerindo que essas variáveis amenizam os efeitos negativos em episódios de insatisfação devido às consequências positivas do controle apresentadas pela literatura. Já a falta de interação entre o controle comportamental (i.e., coprodução) e controle de decisão (i.e., opção de reembolso), que indicaria um papel de moderação da opção de reembolso na relação entre coprodução e intenções comportamentais, parece seguir o mesmo caminho dos resultados encontrados por Mills e Krantz (1979). Em seu experimento no contexto de doação de sangue, eles encontraram um efeito insignificante da interação entre controle cognitivo - nesse caso, dar informação sobre o evento estressante - e controle de decisão - dar a opção de escolher qual braço seria usado para se fazer a doação - na redução do estresse do doador, enquanto o efeito individual de cada controle foi significativo. Ambos os resultados (de Mills e Krantz, 1979 e do presente estudo) parecem indicar que o controle de decisão funciona de forma independente, não interagindo

\section{Gráfico 3 - Intenção de recompra como uma função da coprodução e da opção de reembolso no estudo 2}

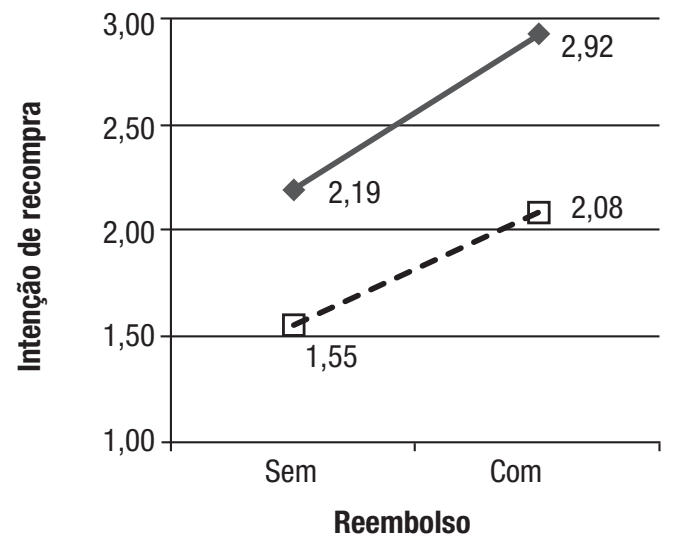

$\multimap$ Coprodução

-も- Sem coprodução com os outros dois tipos de controle, corroborando os achados de Cornelius e Averill (1980).

\section{CONCLUSÕES}

Este trabalho investigou os efeitos da coprodução (controle comportamental), da informação (controle cognitivo) e da opção de reembolso (controle de decisão) nas intenções de recompra e de boca-a-boca positivo após um episódio de compra insatisfatório. Os resultados obtidos indicam que coprodução, opção de reembolso e informação - esta última quando associada à coprodução - podem elevar as intenções comportamentais positivas em relação à empresa após um resultado negativo, amenizando possíveis efeitos prejudiciais à sua imagem e às vendas futuras. A literatura aponta para a escassez de pesquisas empíricas sobre coprodução (MEUTER e outros, 2005) e nenhum trabalho sobre os efeitos da coprodução nas intenções comportamentais foi encontrado, fato que ressalta $\mathrm{O}$ ineditismo das contribuições deste estudo.

O fato de essas três variáveis que representam os tipos de controle definidos por Averill (1973) ameni-

\section{Gráfico 4 - Intenção de boca-a-boca como uma função da coprodução e da opção de reembolso no estudo 2}

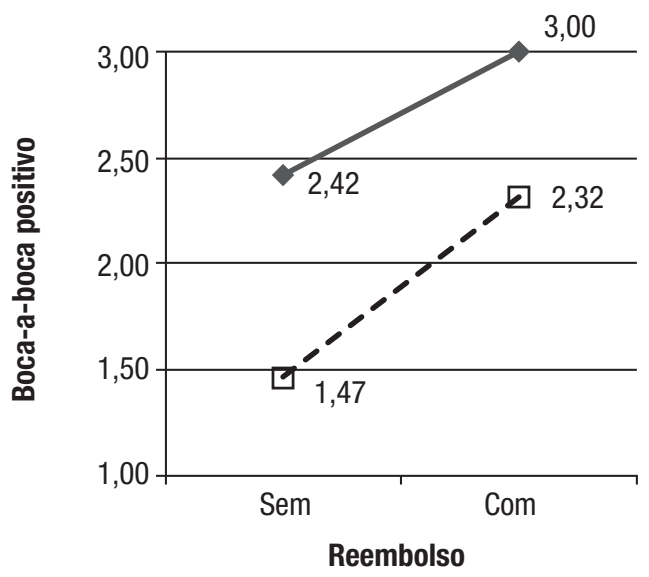


zarem os impactos negativos da insatisfação nas intenções comportamentais do consumidor vai ao encontro de resultados encontrados na literatura, que apontam que o controle tem efeitos positivos nas respostas comportamentais do consumidor (HUI e BATESON, 1991) e na satisfação tanto em experiências do consumidor (CHANG, 2008) quanto em outras esferas da vida (GREENBERGER e outros, 1989; BOND e BUNCE, 2003), e que a satisfação está positivamente relacionada às intenções comportamentais (CRONIN, BRADY, HULT, 2000; LADHARI, 2007, WESTBROOK, 1997). A literatura existente e os resultados dos estudos conduzidos aqui mostram que o controle do processo de produção e de compra, devido aos efeitos positivos já mencionados, pode levar a maiores níveis de intenções comportamentais do consumidor em episódios de insatisfação.

Coprodução, informação e reembolso são ferramentas que as empresas podem gerenciar para captar e reter clientes. A principal contribuição desta pesquisa é a investigação das formas como as empresas podem organizar seu processo produtivo para reduzir os efeitos negativos no comportamento do consumidor após uma insatisfação com a compra, independentemente se essas empresas ofertam serviços ou bens físicos.

A falta de suporte para as hipóteses H2a e H2b, que se referem à influência da informação nas intenções de recompra e de boca-a-boca positivo, sugere que informação não tem efeitos sobre as intenções comportamentais após um resultado insatisfatório quando oferecida sozinha. No entanto, os resultados referentes à interação entre coprodução e nível de informação disponível, que dão suporte às hipóteses H4a e H4b, indicam que o efeito positivo da coprodução nas intenções comportamentais somente é observado quando a participação do consumidor no processo de produção do produto está aliada a uma certa quantidade de informação disponível pela empresa. Isso porque um consumidor pode não se sentir confortável em participar do processo de produção se não receber as informações necessárias para desempenhar as atividades requeridas da maneira correta, uma vez que o controle comportamental na ausência de informação aumentaria o estresse (CORNELIUS e AVERILL, 1980). Assim, fornecer informações relevantes em situações nas quais as empresas permitem a coprodução é altamente desejável, porque parece fazer os consumidores sentirem-se mais capazes de coproduzir, mais no controle da situação e mais satisfeitos com o processo. A ideia de que é preciso oferecer informação suficiente ao consumidor para que o efeito positivo da coprodução se concretize parece ainda mais verdadeira em contextos de serviços, em que, por se tratar de ofertas baseadas em atributos de experiência (ZEITHAML, 1981), isso é, atributos que poderão ser avaliados apenas após a compra, o risco torna-se maior, assim como a assimetria de informação entre compradores e vendedores, que ocorre quando diferentes partes de uma transação têm acesso a quantidades distintas de informação (MOLHO, 1997), na maioria dos casos, o vendedor tendo mais informação que o comprador. A disponibilidade de informação ajudaria, então, a reduzir esse risco e essa assimetria de informações, construindo um cenário propício para que a participação do consumidor no processo de produção da oferta seja bem-sucedida.

A ausência do efeito interativo de coprodução e opção de reembolso, e a confirmação das hipóteses H3a e H3b, referentes ao efeito direto da opção de reembolso nas intenções comportamentais, mostram que a opção de reembolso pode influenciar positivamente as intenções comportamentais após uma compra insatisfatória, mas oferecê-la em conjunto com a coprodução não eleva ainda mais tais intenções do que oferecer coprodução sem reembolso. Consumidores tendem a se comportar de maneira mais positiva quando possuem escolha de reembolso do que quando não possuem essa escolha, porque tal escolha parece levar a maior controle, de maneira semelhante a estudos que encontraram relação positiva entre escolha e controle (CHANG, 2008; HUI e BATESON, 1991; MILLS e KRANTZ, 1979), e menor risco na compra, pois o consumidor pode devolver o produto e receber seu dinheiro de volta se não estiver satisfeito. Uma explicação para a opção de reembolso não ter elevado ainda mais as intenções comportamentais quando oferecida junto com a coprodução pode estar no fato de que o reembolso não faz o consumidor sentir-se mais capaz de desempenhar as tarefas exigidas pela coprodução, ao contrário do que se espera no caso da combinação entre coprodução e informação. Sugere-se que futuras pesquisas investiguem de forma mais profunda a interação entre os controles comportamental e de decisão.

Ainda, podem ser objeto de futuras pesquisas as relações entre as variáveis deste estudo em situações de compras satisfatórias. Em princípio, poderia ser esperado como lógica e natural a mesma relação causal positiva das variáveis independentes nas intenções de recompra e de boca-a-boca positivo, em situações nas quais o cliente ficou satisfeito. Porém, uma revisão da literatura poderia indicar outro caminho. Bendapudi e Leone (2003), por exemplo, encontraram evidências de 
que, em situações nas quais o consumidor obtém os resultados esperados, o nível de satisfação não é diferente entre consumidores que coproduziram e consumidores que não coproduziram. A lógica que permeia esse resultado é que, segundo a teoria da atribuição, as pessoas são mais prováveis de se engajar em um pensamento de atribuição (de causa e efeito), quando confrontadas com o inesperado. Quando coisas esperadas acontecem, há um incentivo menor e, consequentemente, menos esforço em se dar conta sobre quem é responsável por cada parte da tarefa (WEINER, 1985). Assim, quando o resultado é o esperado, não são esperadas diferenças na satisfação do consumidor com a empresa quando o consumidor participa da produção, comparando-se a quando ele não participa (BENDAPUDI e LEONE, 2003). Seguindo o mesmo raciocínio, poderiam ser esperadas intenções comportamentais similares entres esses dois grupos de consumidores (os que coproduzem e os que não coproduzem).

Como limitação desse trabalho convém destacar que, apesar da busca pela equivalência entre os cenários, onde se procurou manipular apenas as variáveis consideradas independentes, mantendo o restante da descrição semelhante, algumas variáveis que poderiam influenciar no estudo não foram mensuradas, como as emoções dos participantes em relação às situações descritas (e.g., raiva e decepção), e sua percepção sobre o comportamento (e.g., boa vontade ou simpatia) do funcionário no cenário descrito. Futuros estudos poderão perpassar essas limitações e aumentar ainda mais a validade interna do experimento coletando pensamentos relacionados aos estímulos, para verificar se as descrições usadas estão realmente manipulando apenas aquele fenômeno que se pretende manipular.

Outra limitação a destacar refere-se ao desenho do segundo experimento, que contou com a manipulação de apenas uma das variáveis manipuladas no primeiro experimento. Enquanto o primeiro experimento contou com a manipulação da coprodução e da informação, no segundo experimento optou-se por manter a coprodução como variável manipulada - devido ao efeito significativo que essa variável teve sobre as variáveis dependentes, aos indícios de que o controle comportamental é mais eficiente que outros tipos de controle (CORNELIUS e AVERILL, 1980) e ao fato de ser considerado o protótipo de controle (SKINNER, 1996) - adicionando a opção de reembolso como variável manipulada no lugar da informação, uma vez que esta não apresentou efeito principal sobre as variáveis dependentes. A intenção de trabalhar com esses dois desenhos experimentais foi possibilitar a investigação de três tipos de controle em dois tipos de oferta, a de um serviço e a de um bem físico, ao mesmo tempo em que se procurou manter certa parcimônia no número de fatores manipulados. A forma como ambos os experimentos foram estruturados possibilitou tal investigação, porém se as três variáveis representantes dos tipos de controle tivessem sido incluídas no segundo experimento a investigação do efeito de interação da coprodução e da informação nas intenções comportamentais poderia ter sido aprofundada. Assim, sugere-se que estudos futuros considerem explorar essas três variáveis em um mesmo experimento.

\section{NOTA DA REDAÇÃO}

Este artigo foi apresentado no XXXV Encontro da Associação Nacional de Pós-Graduação e Pesquisa em Administração (EnANPAD) realizado em 2011 e convidado a participar do processo de avaliação double blind review da RAE pela Diretoria da ANPAD.

\section{REFERÊNCIAS}

ATHANASSOPOULOS, A; GOURNARIS, S; STATHAKOPOULOS, V. Behavioural responses to customer satisfaction: an empirical study. European Journal of Marketing, v. 35, n. 5/6, p. 687-707, 2001.

AVERILL, J. R. Personal control over aversive stimuli and its relationship to stress. Psychological bulletin, v. 80, n. 4, p. 286-303, 1973.

BATESON, J. E. G. Perceived Control and the Service Encounter. In: CZEPIEL, J. A; SOLOMON, M. R; SURPRENANT, C. F. (Ed) The service encounter. Massachusetts / Toronto: Lexington Books, 1985. p. 67-82.

BENDAPUDI, N; LEONE, R. P. Psychological implications of customer participation in co-production. Journal of Marketing, v. 67, n. 1, p. 14-28, 2003.

BOND, F. W; BUNCE, D. The role of acceptance and job control in mental health, job satisfaction, and work performance. Journal of Applied Psychology, v. 88, n. 6, p. 1057-1067, 2003. 
BOTTI, S; MCGILL, A. L. When choosing is not deciding: the effect of perceived responsibility on satisfaction. Journal of Consumer Research, v. 33, n. 2 , 211-219, 2006.

BOTTI, S; BRONIARCZYK, S; HÄUBL, G; HILL, R; HUANG, Y; KAHN, B; KOPALLE, P; LEHMANN, D; URBANY, J; WANSINK, B. Choice under restrictions. Marketing Letters, v. 19, n. 3-4, p. 183-199, 2008.

BURGER, J. M. Negative Reactions to Increases in Perceived Personal Control. Journal of Personality and Social Psychology, v. 56, n. 2, p. 246-256, 1989.

CHAN, K. W; YIM, C. K. (Bennett); LAM, S. S. K. Is customer participation in value creation a double-edged sword? Evidence from professional financial services across cultures. Journal of Marketing, v. 74, n. 3, p. 48-64, 2010.

CHANG, C. C. Choice, perceived control, and customer satisfaction: the psychology of online service recovery. Cyberpsychology \& Behavior, v. 11, n. 3, p. 321-328, 2008.

CHARMS, R. de. Personal causation. New York: Academic Press, 1968.

CORNELIUS, R; AVERILL, J. R. The influence of various types of control on psychophysiological stress reactions. Journal of Research in Personality, v. 14, n. 4, p. 503-517, 1980 .

CRONIN, J. J. Jr; BRADY, M. K; HULT, G. T. M. Assessing the effects of quality, value, and customer satisfaction on consumer behavioral intentions in service environments. Journal of Retailing, v. 76, n. 2, p. 193-218, 2000.

DHOLAKIA, N; ZWICK, D; DENEGRI-KNOTT, J. Technology, Consumers, and Marketing Theory. In: MACLARAN, P; SAREN, M; STERN, B; TADAJEWSKY, M. (Ed) Marketing Theory, 1. ed. London: Sage, 2010.

ERTIMUR, B. The role of perceived control in co-production. European Advances in Consumer Research, v. 8, p. 334-35, 2008.

ETGAR, M. A descriptive model of the consumer co-production process. Journal of the Academy of Marketing Science, v. 36, n. 1, 97-108, 2008.

FARANDA, W. T. A scale to measure the cognitive control form of perceived control: construction and preliminary assessment. Psychology \& Marketing, v. 18, n. 12, p. 12591281, 2001.

FERNANDES, D; SANTOS, C. As consequências comportamentais da insatisfação dos clientes. Revista de Administração Contemporânea, Edição Especial, p. 147-174, 2008.

GREENBERGER, D; STRASSER, S; CUMMINGS, L; DUNHAN, R. The impact of personal control on performance and satisfaction. Organizational Behaviour and Human Decision Processes, v. 43, n. 1, p. 29-51, 1989.

HOLT, D. B. How consumers consume: a typology of consumption practices. Journal of Consumer Research, v. 22, n. 1, p. 1-16, 1995.

HUEFNER, J; HUNT, K. Consumer retaliation as a response to dissatisfaction. Journal of Consumer Satisfaction, Dissatisfaction and Complaining Behavior, v. 13, p. 61-82, 2000.

HUi, M. K; BATESON, J. E.G. Perceived control and the effects of crowding and consumer choice on the service experience. Journal of Consumer Research, v. 18, n. 2, p. 174-84, 1991.

HUI, M. K; TOFFOLI, R. Perceived control and consumer attribution for the service encounter. Journal of Applied Social Psychology, v. 32, n. 9, p. 1825-44, 2002.

HUNT, D; ONETO, S. G; VARCA, P. Satisfaction in the context of customer co-production. In: DAHL, D. W; JOHAR, G. V; van OSSElAER, S. M. J. (Ed) Advances in Consumer Research, v. 38. Duluth, MN: Association for Consumer Research, 2010.

LADHARI, R. The effect of consumption emotions on satisfaction and word-of-mouth communications. Psychology E Marketing, v. 24, n. 12, p. 1085-1108, 2007.

LANGER, E. J. The illusion of control. Journal of Personality and Social Psychology, v. 32 n. 2, p. 311-328, 1975.

LUSCH, R. F; VARGO, S. L; O'BRIEN, M. Competing through service: insights from service-dominant logic. Journal of Retailing, v. 83, n. 1, p. 5-18, 2007.

MEUTER, M. L; BITNER, M. J; OSTROM, A. L; BROWN, S. W. Choosing among alternative service delivery modes: an investigation of customer trial of self-service technologies. Journal of Marketing, v. 69, n. 2, p. 61-83, 2005. 
MILLS, R. T; KRANTZ D. S. Information, choice, and reactions to stress: a field experiment in a blood bank with laboratory analogue. Journal of Personality and Social Psychology, v. 37, n. 4, p. 608-620, 1979.

MOLHO, I. The Economics of Information: lying and cheating in markets and organizations. Oxford: Blackwell Publishing, 1997.

ORDANINI, A; PASINI, P. Service co-production and value cO-creation: the case for a service-oriented architecture (SOA). European Management Journal, v. 26, n. 5, p. 289-297, 2008.

PRAHALAD, C.K; RAMASWAMY, V. Co-creation experiences: the next practice in value creation. Journal of Interactive Marketing, v. 18, n. 3, p. 5-14, 2004.

SHARMA, S; DURAND, R. M; GUR-ARIE, O. Identification and Analysis of Moderator Variables. Journal of Marketing Research, v. 18, n. 3, p. 291-300, 1981.

SINGH, J. Consumer complaint intentions and behavior: definitional and taxonomical. Journal of Marketing, v. 52, n. 1, p. 93-107, 1988.

SKINNER, E. A. A guide to constructs of control. Journal of Personality and Social Psychology, v. 71, n. 3, p. 549571, 1996.

STAUB, E; TURSKY, B; SCHWARTZ, G. E. Self-control and predictability: their effects on reactions to aversive stimulation. Journal of Personality and Social Psychology, v. 18, n. 2, p. 157-162, 1971.

THOMPSON, S. C. Will it hurt less if I can control it? A complex answer to a simple question. Psychological Bulletin, v. 90, n. 1, p. 89-101, 1981.

VAN RAAIJ, W. F., PRUYN Ad. Th. H. Customer control and evaluation of service validity and reliability. Psychology $\mathrm{E}$ Marketing. v. 15, n. 8, p. 811-832, 1998.

VAN ROMPAY, T. J. L; GALETZKA, M; PRUYN, A. T. H; GARCIA, J. M. Human and spatial dimensions of retail density: revisiting the role of perceived control. Psychology \& Marketing, v. 25, n. 4, p. 319-335, 2008.

VARGO, S. L; LUSCH, R. F. Service-dominant logic: continuing the evolution. Journal of the Academy of Marketing Science, v. 36, n. 1, p. 1-10, 2008.
WEINER, B. An attributional theory of achievement motivation and emotion. Psychological Review, v. 92, n. 4, p. 548-573, 1985.

WESTBROOK, R. A. Product/consumption-based affective responses and postpurchase processes. Journal of Marketing Research, v. 24, n. 3, p. 258-270, 1987.

WHITE, R. W. Motivation reconsidered: The concept of competence. Psychological Review, v. 66, p. 297-333, 1959.

WITT, L. A; ANDREWS, M. C; KACMAR, K. M. The role of participation in decision-making in the organizational politics-job satisfaction relationship. Human Relations, v. 53, n. 3, p. 341-358, 2000.

ZEITHAML, V. How consumer evaluation processes differ between goods and services. In: DONNELLY, J. A.; GEORGE, W. R. (Ed) Marketing of Services. Chicago: American Marketing Association, 1981, p. 186-190.

ZEITHAML, V. A; BERRY, L; PARASURAMAN, A. The behavioral consequences of service quality. Journal of Marketing, v. 60, n. 2, p. 31-46, 1996.

ZHANG, S; FITZSIMONS, G. J. Choice-process satisfaction: the influence of attribute alignability and option limitation. Organizational Behavior and Human Decision Processes, v. 77, n. 3. p. 192-214, 1999. 